\title{
Фотопроводимость монокристаллов слоистого полупроводника p-GaSe, легированного редкоземельными элементами, и многодиапазонный фотоприемник света на их основе
}

\author{
(C) А.Ш. Абдинов ${ }^{1}$, Р.Ф. Бабаева ${ }^{2}$ \\ ${ }^{1}$ Бакинский государственный университет, \\ Az1145 Баку, Республика Азербайджан \\ ${ }^{2}$ Азербайджанский государственный экономический университет, \\ Az1001 Баку, Республика Азербайджан \\ E-mail: abdinov-axmed@yandex.ru, babaeva-rena@yandex.ru
}

Поступила в Редакцию 26 апреля 2018 г.

В окончательной редакции 11 декабря 2021 г.

Принята к публикации 15 декабря 2021 г.

\begin{abstract}
Экспериментально исследованы основные характеристики собственной фотопроводимости и спектры отрицательной фотопроводимости, индуцированной примесной фотопроводимости, инфракрасного гашения собственной фотопроводимости в легированных редкоземельными элементами (гадолинием и диспрозием) при $N=0-10^{-1}$ ат\% монокристаллах $p$-GaSe. Показано, что нестабильность и невоспроизводимость фотоэлектрических характеристик этого полупроводника обусловлены флуктуацией электронного потенциала, связанной с наличием случайных макроскопических дефектов. Зависимость фотоэлектрических параметров и характеристик от $N$, а также обеспечение высокой степени их стабильности и воспроизводимости при $N \approx 10^{-1}$ ат\% связаны соответствующими изменениями флуктуаций электронного потенциала и доли ковалентной связи между слоями в зависимости от $N$. Показана возможность создания многодиапазонного фотоприемника света со стабильными, воспроизводимыми параметрами и характеристиками на основе $p$-GaSe, легированного редкоземельными элементами при $N=10^{-2}-10^{-1}$ ат\%.
\end{abstract}

Ключевые слова: оптоэлектроника, межслойная связь, спектральное распределение, кинетика, макроскопические дефекты, матрица, стабильность, воспроизводимость.

DOI: 10.21883 /FTP.2022.05.52346.8902

\section{1. Введение}

Слоистая кристаллическая структура, высокая химическая стойкость, возможность расслаивания до наноразмерных слоев с атомарно-зеркальной поверхностью делают фоточувствительные монокристаллы $p$-GaSe перспективным материалом для оптоэлектроники [1-3]. Однако слабая межслойная связь в этом полупроводнике приводит к низкой механической прочности и твердости, которые, вызывая случайное дефектообразование, заметно затрудняют изготовление фотоэлектрических функциональных элементов высокого качества на основе его монокристаллов. Повышение механической прочности и твердости GaSe возможно при легировании [4,5], которое непосредственно может разрешить эту проблему.

В данной работе для целенаправленного управления влиянием случайных дефектов на фотоэлектрические свойства этого полупроводника путем легирования, а также выявления новых возможностей его практического применения экспериментально исследована фотопроводимость монокристаллов $p$-GaSe, легированных редкоземельными элементами (Р3Э) - гадолинием $(\mathrm{Gd})$ и диспрозием (Dy).

\section{2. Методика эксперимента и образцы}

Исследуемые образцы изготовлены путем простого расслаивания из выращенных видоизмененным методом
Бриджмена монокристаллических слитков $p$-GaSe, легированных редкоземельными элементами $(p-\mathrm{GaSe}\langle\mathrm{P} 3 Э\rangle)$, с содержанием введенной примеси $N=0,10^{-5}, 5 \cdot 10^{-5}$, $10^{-4}, 5 \cdot 10^{-4}, 10^{-3}, 5 \cdot 10^{-3}, 10^{-2}, 10^{-1}$ ат\%. Выбор в качестве примесей Dy и Gd был вызван индивидуальными физическими особенностями этих химических элементов и возможностями технологии при легировании ими монокристаллов $p$-GaSe [6,7].

Структура, фазовый и элементный состав использованных слитков, состояние поверхности исследуемых образцов вдоль плоскости (001) кристалла были определены путем проведения комплексных термографических, рентгенографических и микроскопических анализов при помощи установок типа ДСК-910, ADVNCE-8D, SINTECP 2, ДРОН-4-07 с использованием $\mathrm{Cu} K_{\alpha}$-излучения при шаге 0.05 и диапазоне углов $8-13.5^{\circ}$, SEM фирмы Zeiss с энергодисперсионным анализатором. Установлено, что использованный материал является гомогенным по химическому составу и кристаллической структуре, имеет монокристаллическую структуру, относится к $\varepsilon$-политипу с пространственной группой $D_{1 h}^{1}(P \overline{6})$ и периодами элементарной ячейки $a \approx 3.744 \AA, c \approx 15.902 \AA[8]$. Фазы замещения селенидов и оксидов РЗЭ в этих слитках, а также неоднородности и посторонние фазы на поверхности исследуемых образцов не обнаружены [6,7]. 


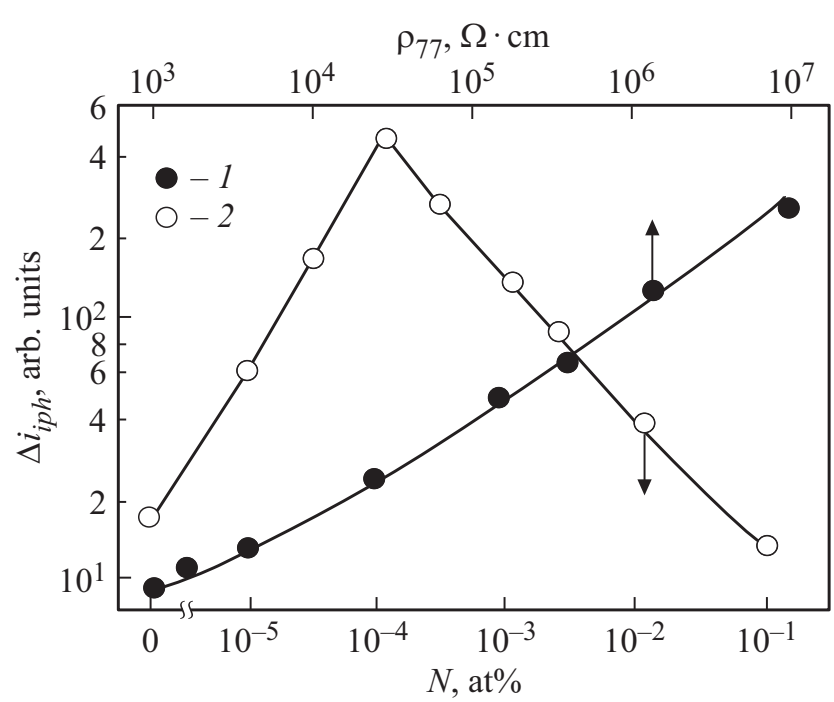

Рис. 1. Зависимости величины собственной фотопроводимости от исходной величины удельного темнового сопротивления в нелегированных (кривая 1) и содержания введенной примеси (кривая 2) в легированных РЗЭ кристаллах $p$-GaSe при $77 \mathrm{~K} . I_{\text {own }}=0.1 I_{\text {own.m }} ; \lambda_{\text {own }}=0.580$ мкм $($ собственный - own $)$.

В диапазоне температур $77 \leq T \leq 300 \mathrm{~K}$, длин волн $0.200 \leq \lambda \leq 3.000$ мкм и при интенсивностях света $I \leq 4 \cdot 10^{2}$ Лк при помощи экспериментальной установки, собранной на базе двух монохроматоров типа МДР-12 [9], методом стационарной фотопроводимости [10] снимались спектральное распределение, люксамперная характеристика (ЛАХ) и кинетика фотопроводимости. При измерениях ток протекал вдоль естественных слоев кристалла, а световой поток был направлен перпендикулярно к ним.

\section{3. Экспериментальные результаты}

Установлено, что в области температур ниже $250 \mathrm{~K}$ удельные темновые сопротивления различных образцов нелегированных $(N=0$ ат\%) кристаллов различаются и при $77 \mathrm{~K}$ изменяются в интервале $10^{3} \leq \rho_{77} \leq 10^{7}$ Ом·см. В этой области температур при освещенностях $I \leq 35-40$ Лк с ростом значения $\rho_{77}$ величина собственной фотопроводимости $\left(\Delta i_{i p h}=\left(i_{s v l k}-i_{s v d c}\right) / i_{s v d c}\right.$, где $i_{s v l c}$ и $i_{s v d c}-$ стационарные значения светового и темнового тока соответственно) увеличивается (рис. 1, кривая 1), ее спектральное распределение расширяется в сторону более длинных волн (рис. 2, кривые 1 и 5), в самом начале ЛАХ наблюдается степенной участок $\Delta i_{i p h} \sim I^{\alpha}$ с $\alpha \geq 2$ (рис. 3, кривые 1 и 2), а кинетика становится медленно релаксирующей (рис. 4, кривые 1 и 2).

В легированных же кристаллах $p$-GaSe величина и характеристики собственной фотопроводимости от химической природы введенной примеси не зависят, а с изменением содержания введенной примеси при температурах $T \leq 250 \mathrm{~K}$ и освещенностях $I \leq 35-40$ Лк немонотонно отклоняются от имеющих место в нелегированных образцах с наименьшим $\rho_{77}$ (рис. 1, кривая 2 и рис. 2-4, кривые 3 и 4). Наибольшее отклонение наблюдается при $N \approx 5 \cdot 10^{-4}$ ат\%, а наименьшее - при $N \approx 10^{-1}$ ат\%. Образцы кристаллов $p$-GaSe $\langle$ Р3Э $\rangle$ с $N \approx 10^{-1}$ ат\% обладают также высокой стабильностью и воспроизводимостью фотоэлектрических параметров и характеристик. С повышением температуры влияние легирования на фотопроводимость кристаллов $p$-GaSe постепенно ослабляется.

При $N \approx 10^{-2}-10^{-1}$ ат\% спектральное распределение собственной фотопроводимости $\left(\Delta i_{i p h}\right)$ монокристаллов

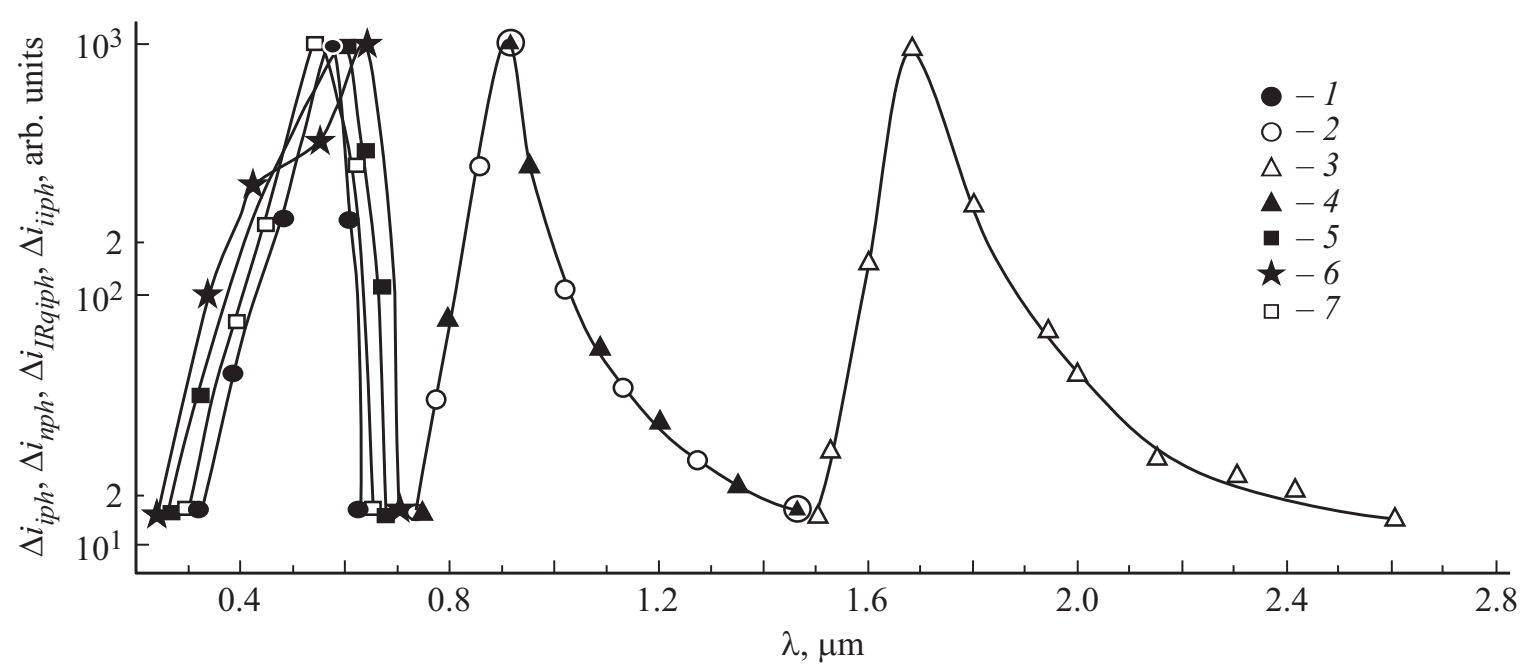

Рис. 2. Спектральное распределение собственной фотопроводимости (кривые 1,5-7), отрицательной фотопроводимости (кривая 2), ИК-гашения собственной фотопроводимости (кривая 3) и индуцированной примесной фотопроводимости (кривая 4 ) в кристаллах $p$-GaSe $\langle$ РЗЭ $\rangle$ при $77 \mathrm{~K} . N$, ат\%: $1-5-0,6-10^{-4}, 7-10^{-1} ; \rho_{77}$, Ом · см: $1-4-3 \cdot 10^{3}, 5-10^{7}$. 
$p$ - $\mathrm{GaSe}\langle$ РЗЭ $\rangle$ совпадает со спектром чувствительности человеческого глаза $\left(V_{\text {sshe }}\right)$ (рис. 5).

В кристаллах $p$-GaSe при $T \geq 200 \mathrm{~K}$ в диапазоне $\lambda \approx 0.750-1.450$ мкм наблюдаются также отрицатель-

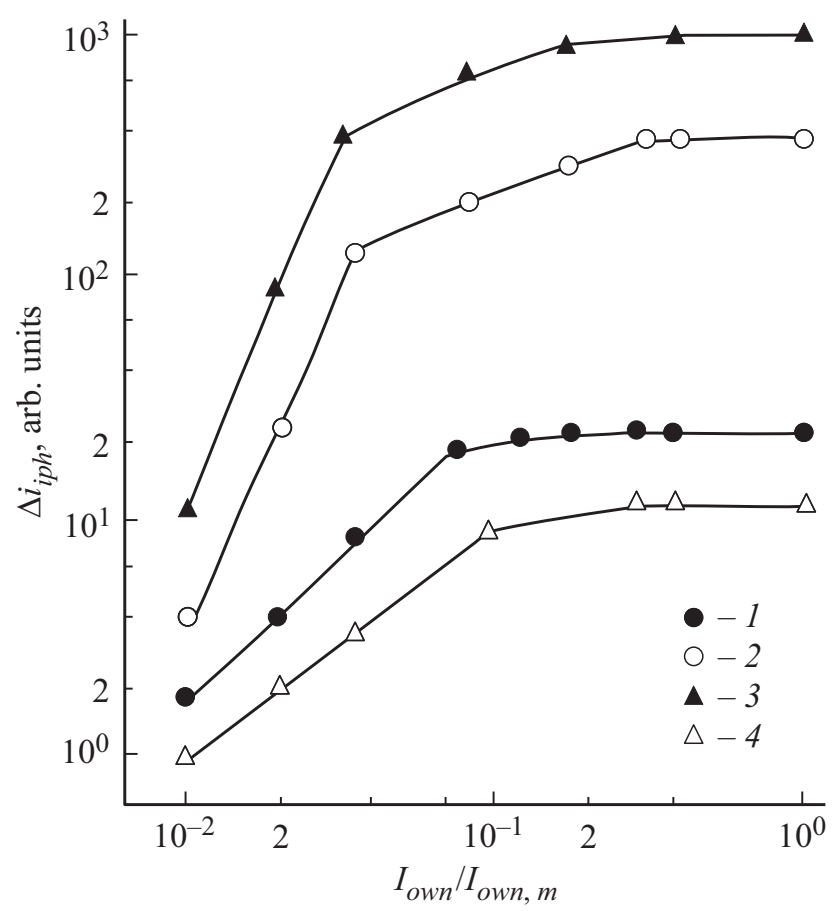

Рис. 3. Люкс-амперная характеристика собственной фотопроводимости в кристаллах $p$-GaSe $\langle$ Р3Э $\rangle$ при $77 \mathrm{~K} . N$, ат\%: $1,2-0 ; 3-10^{-4} ; 4-10^{-1} ; \lambda_{\text {own }}=0.580$ мкм; $\rho_{77}$, Ом $\cdot$ см: $1-5 \cdot 10^{3}, 2-10^{7}$.

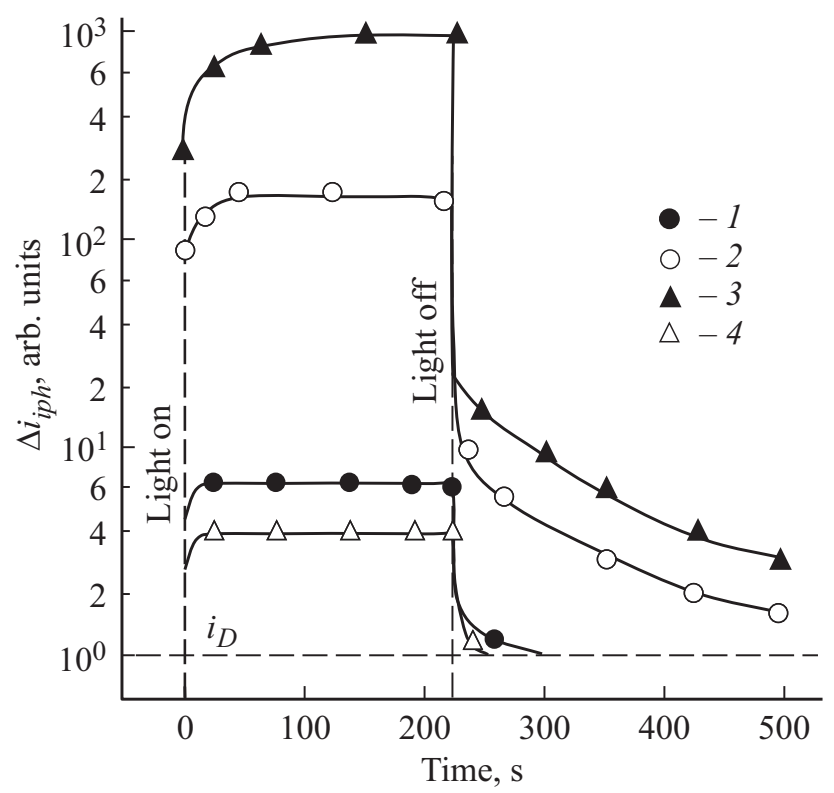

Рис. 4. Кинетика собственной фотопроводимости в кристаллах $p$ - $\mathrm{GaSe}\langle$ Р3Э $\rangle$ при $77 \mathrm{~K} . N$, ат\%: $1,2-0 ; 3-10^{-4}$, $4-10^{-1} ; \lambda_{\text {own }}=0.580 \mathrm{м \kappa M}, I_{\text {own }}=0.15 I_{\text {own.m }} ; \rho_{77}, \mathrm{OM} \cdot \mathrm{cм}$ : $1-5 \cdot 10^{3}, 2-10^{7}$.

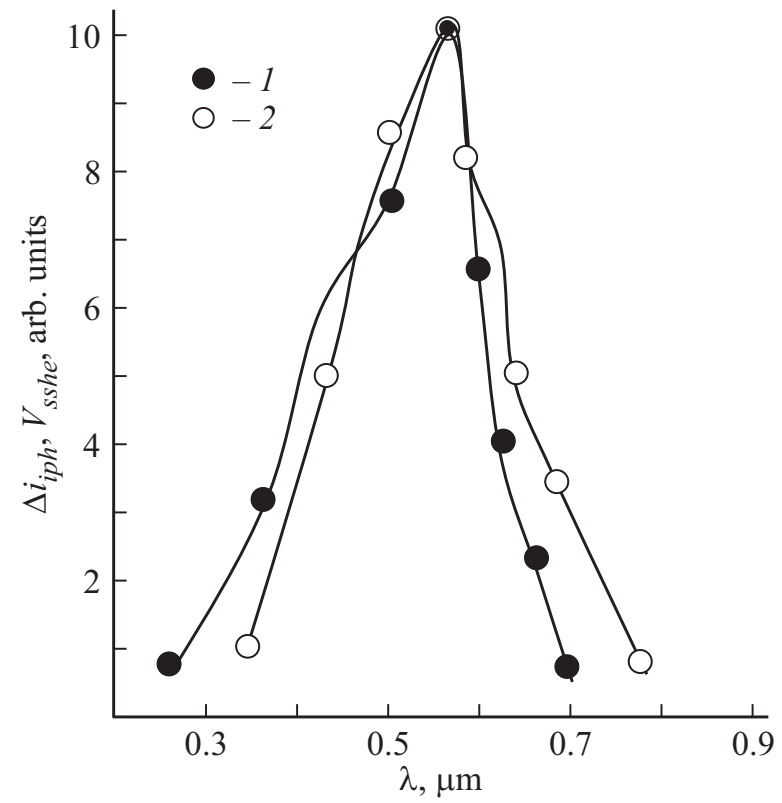

Рис. 5. Спектральное распределение собственной фотопроводимости в кристаллах $p$-GaSe $\langle$ Р3Э $\rangle$ с $N=10^{-1}$ ат\% (кривая 1) при $77 \mathrm{~K}$ и чувствительности человеческого глаза (кривая 2).

ная фотопроводимость $\left(\Delta i_{n p h}\right)$ и ИК-гашение собственной фотопроводимости $\left(\Delta i_{\text {IRqiph }}\right)$, а в диапазоне $\lambda \approx 1.500-2.600$ мкм еще и индуцированная примесная фотопроводимость $\left(\Delta i_{i i p h}\right)$ (рис. 1 , кривые 2,3 и 4 соответственно). Величины и ход основных характеристик $\Delta i_{n p h}, \Delta i_{\text {IRqiph }}, \Delta i_{i i p h}$ не зависят от легирования, но в образцах $p$-GaSe $\langle\mathrm{P} 3 Э\rangle$ с $N \approx 10^{-2}-10^{-1} \mathrm{aT} \%$ становятся наиболее стабильными и воспроизводимыми. Эти примесные фотоэлектрические явления, кроме расширения спектрального диапазона фоточувствительности кристаллов $p$-GaSe до $\sim 2.600$ мкм, придают им возможность реагировать на слабое ИК-излучение с $\lambda=0.800-2.600$ мкм на фоне видимого света с большой интенсивностью.

Установленные экспериментальные данные свидетельствуют о возможности целенаправленного управления стабильностью и воспроизводимостью фотоэлектрических параметров и характеристик $p$-GaSe путем легирования Р3Э (Dy и Gd), а также создания на основе монокристаллов $p$-GaSe $\langle$ Р3Э $\rangle$ с $N=10^{-1}$ ат\% высококачественного фотоприемника света для диапазонов оптического излучения $\lambda \approx 0.350-0.630, \approx 0.750-1.450$ и $\approx 1.500-2.600$ мкм.

\section{4. Обсуждение}

На основе статистического анализа экспериментальных результатов установлено, что в образцах нелегированных $(N=0$ aт\%) кристаллов $p$-GaSe c $\rho_{77} \leq 5 \cdot 10^{3} \mathrm{OM} \cdot \mathrm{cm}$ характеристики собственной фотопроводимости удовлетворительно объясняются на ос- 
нове модели кристаллического полупроводника с различными типами центров захвата, рекомбинации и прилипания. Однако обнаруженные при $T \leq 250 \mathrm{~K}$ и $I \leq 35-40$ Лк зависимости величины (рис. 1), спектрального распределения (рис. 2), ЛАХ (рис. 3), кинетики (рис. 4) собственной фотопроводимости от значений $\rho_{77}$ в образцах нелегированных и от значений $N$ в образцах легированных кристаллов на основе этой модели не объясняются. Для их объяснения, кроме существования различного типа центров захвата, рекомбинации и прилипания [11], необходимо учитывать также флуктуации электронного потенциала, связанные с наличием случайных макроскопических дефектов (СМД) в исследуемых образцах $[6,7,12]$. Являющиеся однофазными с матрицей $(\mathrm{M})$, эти дефекты возникают при изготовлении образцов вследствие локальных нарушений упорядоченности слоистой структуры из-за слабой межслойной связи [6,7]. Атомы РЗЭ, имеющие очень низкую растворимость при использованных в данной работе технологических режимах и содержаниях примесей, доминирующим образом входят в междоузлия и, благодаря малой электроотрицательности, отдавая свой валентный электрон, легко ионизируются. Далее, ионы РЗЭ под действием внутреннего электрического поля, созданного на границе М-СМД $p^{+}-p$-перехода, скапливаются на СМД и увеличивают их размеры. При малых содержаниях примесей расстояние между областями пространственного заряда (ОПЗ) соседних СМД будет больше длины диффузии $\left(L_{D}\right)$, а следовательно, и длины свободного пробега носителей заряда. При увеличении содержания примеси флуктуации электронного потенциала становятся больше, чем в нелегированных кристаллах, и влияние СМД на фотопроводимость усиливается. С дальнейшим увеличением $N$ расстояние между областями пространственного заряда соседних СМД становится сравнимо с $L_{D}$, а следовательно, и с длиной свободного пробега носителей заряда. При этом ОПЗ соседних СМД начинают постепенно „сплачиваться“ и образцы монокристаллов $p$-GaSe $\langle$ Р3Э $\rangle$ приближаются к характерному для пространственно-однородного кристаллического полупроводника состоянию. Кроме этого, из-за роста доли ковалентной связи между ионами трехвалентного РЗЭ, размещенных в соседних слоях и случайных вакансиях галлия, усиливается также межслойная связь в кристалле. Оба эти процесса в свою очередь приводят к повышению стабильности и воспроизводимости параметров и характеристик фотопроводимости в $p-\mathrm{GaSe}\langle\mathrm{P} 3 Э\rangle$.

В рамках этой модели зависимость спектра собственной фотопроводимости от $\rho_{77}$ в нелегированных и от значений $N$ в легированных образцах объясняется изменением вероятности туннелирования неравновесных носителей заряда через рекомбинационные барьеры на границе М-СМД. Следует отметить, что связанная с туннелированием значительная величина фотопроводимости, несмотря на малую вероятность этого эффекта, обусловлена большим временем существования (до $\sim 10^{3}-10^{5}$ с) неравновесных электронно-дырочных пар, разделенных рекомбинационными барьерами.

\section{5. Заключение}

На основе экспериментального исследования фотопроводимости легированных $\mathrm{P} 3 Э$ ( $\mathrm{Gd}$ и $\mathrm{Dy}$ ) монокристаллов $p$-GaSe установлено, что нестабильность и невоспроизводимость фотоэлектрических параметров и характеристик этого полупроводника обусловлены флуктуацией электронного потенциала, связанного со случайными макроскопическими дефектами. Зависимость фотоэлектрических параметров и характеристик от содержания введенной примеси, а также обеспечение высокой степени их стабильности и воспроизводимости при $N \approx 10^{-1}$ ат\% связаны соответствующими изменениями флуктуаций электронного потенциала и доли ковалентной связи между слоями в зависимости от $N$. Легированные $\mathrm{Gd}$ и Dy c $N \approx 10^{-1} \mathrm{aT} \%$ монокристаллы $p$-GaSe могут быть использованы для создания работающего в диапазонах $0.350 \leq \lambda \leq 0.630,0.750 \leq \lambda \leq 1.450$, $1.500 \leq \lambda \leq 2.600$ мкм многодиапазонного фотоприемника света и фотоприемника света для регистрации слабого ИК-излучения $(0.800 \leq \lambda \leq 2.600$ мкм) на фоне видимого света с высокой интенсивностью.

\section{Конфликт интересов}

Авторы заявляют, что у них нет конфликта интересов.

\section{Список литературы}

[1] E.B. Borisenko, N.N. Kolesnikov, D.N. Borisenko, S.I. Bozhko. J. Cryst. Growth, 316 (1), 20 (2011).

[2] V.V. Atuchin, Y.M. Andreev, K.A. Kokh, G.V. Lanskii, A.V. Shaiduko, T.I. Izaak, V.A. Svetlichnyi. Proc. SPIE, 8772, 87721Q-3 (2013).

[3] P.J. Ko, A. Abderrahmane, T. Takamura, N.-H. Kim, A. Sandhu. Nanotechnol., 27 (32), 325202 (2016).

[4] Z.S. Feng, Z.H. Kang, F.G. Wu, J.Y. Gao, Y. Jiang, H.Z. Zhang, Y.M. Andreev, G.V. Lanskii, V.V. Atuchin, T.A. Gavrilova. Opt. Express, 16, 9978 (2008).

[5] Z.S. Rak, S.D. Mahanti, K.C. Mandal, N.C. Fernelius. Solid State Commun., 150, 1200 (2010).

[6] А.Ш. Абдинов, Р.Ф. Бабаева. Неорг. матер., 55 (4), 355 (2019). [A.Sh. Abdinov, R.F. Babaeva. Inorg. Mater., 55 (4), 355 (2019)].

[7] А.Ш. Абдинов, Р.Ф. Бабаева. Изв. вузов. Физика, 61 (729), 102 (2018). [A.Sh. Abdinov, R.F. Babaeva. Russian Phys. J., $61(9), 1667$ (2019)].

[8] A. Kuhn, A. Chevy, R. Chevalier. Phys. Status Solidi A, 31, 469 (1975).

[9] А.Ш. Абдинов, Р.Ф. Бабаева. Неорг. матер., 55 (8), 806 (2019). [A.Sh. Abdinov, R.F. Babaeva. Inorganic materials, 55 (8), 758 (2019)].

[10] С.М. Рывкин. Фотоэлектрические явления в полупроводниках (М., Наука, 1963) гл. 2, с. 37. 
[11] А.Ш. Абдинов, В.К. Мамедов, Эль.Ю. Салаев. ФТП, 14 (4), 754 (1980).

[12] K.A. Kokh, V.V. Atuchin, T.A. Gavrilova, A. Kozhukhov, E.A. Maximovsky, L.D. Pokrovsky, A.R. Tsygankova, A.I. Saprykin. J. Microscopy, 256 (3), 208 (2014).

Редактор А.Н. Смирнов

\title{
Photoconductivity of the layered semiconductor $p$-GaSe $\langle$ REE $\rangle$ and multiband photoreceiver of light on their basis
}

\author{
A.Sh. Abdinov' ${ }^{1}$, R.F. Babayeva ${ }^{2}$ \\ ${ }^{1}$ The Baku State University, \\ AZ1145 Baku, Azerbaijan, \\ 2 The Azerbaijan State University of Economics \\ (UNEC), \\ AZ1001 Baku, Azerbaijan
}

Abstract The main characteristics of intrinsic photoconductivity and the spectra of negative photoconductivity induced by impurity photoconductivity, infrared quenching of intrinsic photoconductivity in doped REE (gadolinium and dysprosium) at $N=0-10^{-1}$ at $\%, p$-GaSe single crystals are experimentally investigated. It is shown that the instability and irreproducibility of the photoelectric characteristics of this semiconductor are due to fluctuations in the electronic potential associated with the presence of random macroscopic defects. The dependence of photoelectric parameters and characteristics on $N$, as well as ensuring a high degree of their stability and reproducibility at $N \approx 10^{-1}$ at $\%$, are associated with the corresponding changes in the fluctuations of the electronic potential and the fraction of covalent bonds between the layers, depending on $N$. Shown is the possibility of creating a multi-range photodetector of light with stable, reproducible parameters and characteristics based on $p$-GaSe $\langle$ REE $\rangle$ at $N=10^{-2}-10^{-1}$ at $\%$. 